\title{
GERAÇÃO DE MODELO DIGITAL DE TERRENO POR RESTITUIÇÃO AEROFOTOGRAMÉTRICA COM APOIO DE VEÍCULO AÉREO NÃO TRIPULADO DE PEQUENO PORTE: ESTUDO DE CASO NA PEDREIRA DA EMPRESA INCOPEL - ESTÂNCIA VELHA / RS
}

\author{
Marciano Carneiro \\ Rute Henrique da Silva Ferreira' \\ Telmo Fernando Perez de Quadros ${ }^{2}$ \\ Rubens Müller Kautzmann ${ }^{1,2}$
}

Resumo

O interesse em veículos aéreos não tripulados (VANT) tem crescido rapidamente. Os avanços recentes na tecnologia computacional, desenvolvimento de software e sensores sofisticados são os principais motivos do aumento desta tecnologia. Atualmente, o Brasil e outros países trabalham para o desenvolvimento de VANT em diferentes mercados, destacando-se a agricultura de precisão e o mapeamento urbano. O objetivo desta pesquisa é estudar a viabilidade técnica do uso de dados planialtimétricos obtidos a partir de fotografias aéreas digitais tomadas por câmeras a bordo de pequenos VANTs para a geração de modelo digital do terreno (MDT) que seja capaz de dar suporte para o cálculo de volume em cavas de minas. O experimento foi realizado na pedreira da INCOPEL, em Estância Velha, onde foram instalados alvos de controle para a restituição aérea da imagem e alvos amostrais nas sete bancadas da pedreira. Estudos comparativos foram realizados comparando as elevações ortométricas medidas com a técnica GNSS RTK com as medidas no Photogrammetry Software PhotoScan. Também foi avaliada a correlação entre as variáveis experimentais. Constatou-se que a escala encontrada no experimento satisfaz a norma da Comissão Nacional de Cartografia (CONCAR), no que diz respeito às especificações técnicas de dados geoespaciais vetoriais e atende a finalidade de cálculo de volumes em cavas de minas.

Palavras-chave: VANT; Sensoriamento remoto; Avaliação de impactos ambientais; Geodésia.

\section{GENERATION OF DIGITAL MODEL OF LAND BY AEROPHOTOGRAMETRIC RESTITUTION WITH SUPPORT OF SMALL PORTIE AIRCRAFT VEHICLE: CASE STUDY IN INCOPEL QUARRY - ESTÂNCIA VELHA / RS}

\begin{abstract}
The interest in Unmanned Aerial Vehicle (UAV) has been increased very fast. Recent advances in computer technology, software and sophisticated sensors are the main reasons for the increase of this technology. Nowadays, Brazil and other countries work for the development of UAV in different markets, highlighting the precision agriculture and urban mapping. The objective of the this research is to study the technical feasibility of the use of planialtimetric data obtained from digital aerial photographs taken by on board cameras in small UAV for the generation of digital terrain model (DTM) that it is able to give support for the calculation of volume in mine caves. The experiment was done at INCOPEL quarry, in Estância Velha, where it was installed control targets for aerial restitution of the picture and sample targets on the seven workbenches of the quarry. Thus, comparative studies were done between orthometric elevations measured with GNSS RTK technique and the ones that were measured in the Photogrammetry Software PhotoScan. It was also checked the correlation between the experiment variables. It was found that the scale found in the experiment satisfies the National Commission of Cartography (CONCAR) standard, regarding the technical specifications of vector geospatial data and serves the purpose of calculating volumes in open-pit mine.
\end{abstract}

Keywords: UAV; Remote sensing; Environmental impact assessment; Geodesy.

'Centro Universitário La Salle - UNILASALLE, Canoas, RS, Brasil. E-mail: rute.ferreira@unilasalle.edu.br.

${ }^{2}$ Departamento Nacional de Produção Mineral - DNPM, Porto Alegre, RS, Brasil.

2176-1523 (C) 2017 Associação Brasileira de Metalurgia, Materiais e Mineração. Publicado pela ABM. Este é um artigo de acesso aberto distribuído sob os termos da licença Creative Commons CC BY-NC-ND (Attribution-NonCommercial-NoDerivs) - https:// creativecommons.org/licenses/by-nc-nd/4.0\%. 


\section{INTRODUÇÃO}

O interesse por Veículos Aéreos Não Tripulados (VANTs) tem crescido rapidamente. A aerofotogrametria realizada a partir de sensores (especialmente câmeras digitais) colocados a bordo de VANTs, utilizada inicialmente para fins militares, está consolidada para a agricultura de precisão, monitoramento de impactos ambientais, cadastro urbano, sensoriamento remoto, mapeamento de áreas, como o de cavas de minas [ $\mathrm{I}$ ]. O crescimento do uso desta tecnologia se deve a fatores como capacidade de sobrevoar áreas de difícil acesso ou hostis, baixo custo e facilidade de operação, baixo risco de acidentes envolvendo pessoas e utilização em espaços reduzidos com baixa altitude de vôo (grandes escalas). Tais qualidades tornam esta tecnologia atrativa para ser utilizada em áreas de mineração (objeto deste trabalho), onde há cada vez mais preocupações com a segurança do operador do instrumento topográfico e do pessoal auxiliar. Além disso, a utilização de VANTs pode auxiliar nos trabalhos de fiscalização de minas e de barragens. Neste último caso, permite, por exemplo, o imageamento da estrutura da barragem e a geração de mapas de inundação, visando à definição de áreas a serem atingidas em caso de rompimento de barragens. O mapeamento realizado com VANTs na mineração concentra-se na fotogrametria, seja ela de médio, pequeno ou, mais frequente, a de micro levantamentos. A utilização de topografia convencional, imagens de satélites e aerolevantamentos realizados com sensores de grande porte implicam custo elevado dos produtos finais, além de necessitar condições climáticas favoráveis em um longo período de tempo.

O baixo peso dos VANTs traz a preocupação com a estabilidade dos mesmos, especialmente nas decolagens e no enfrentamento de turbulências. Esta estabilidade também é importante no aspecto da qualidade geométrica das aerofotos feitas a partir de câmeras a bordo deles. Alguns trabalhos de pesquisa têm sido direcionados aos aspectos de segurança e estabilidade, como pode ser visto em trabalhos de obtenção de dados dendrométricos de Eucalyptus benthamii Maiden et Cambage nos estudos de Slompo [2] e uso de veículos aéreos não tripulados (VANT) em agricultura de precisão nos estudos de Jorge [I].

A pesquisa realizada se insere no campo da aerofotogrametria. Segundo Brito e Coelho [3] entre os vários objetivos da fotogrametria, um dos principais é a redução do trabalho de campo no levantamento de coordenadas de um ou vários objetos e o tempo de obtenção de tais informações.

O presente trabalho estuda a viabilidade técnica do emprego de dados planialtimétricos resultantes de restituição de aerofotos digitais geradas em câmeras a bordo de VANT de pequeno porte, para geração de modelo digital de terreno (MDT) capaz de dar suporte ao cálculo de volumes em cavas de minas.

Quando se trata de imagens aéreas da superfície terrestre por meio de sensores digitais aerotransportados, o detalhe mais importante para a definição da precisão e acurácia com que se necessita obter o produto cartográfico não é a escala da foto e a altitude de voo, como no caso de câmeras aéreas convencionais, e sim o tamanho ou resolução espacial do pixel no terreno [3]. Esse parâmetro também é conhecido como GSD (Ground Sample Distance) e está para a fotogrametria digital como a escala do voo, calculada como a razão entre a distância focal e a altura de vôo, está para a fotogrametria convencional.

Uma preocupação ao realizar trabalhos com o uso da fotogrametria é a sua acurácia. Segundo a Comissão Nacional de Cartografia (CONCAR), para que um produto digital possa ser aceito como produto de referência do Sistema Cartográfico Nacional (SCN), e consequentemente para a Infraestrutura Nacional de Dados Espaciais (INDE), a exemplo do previsto para o indicador estatístico de qualidade posicional, denominado Padrão de Exatidão Cartográfico (PEC) para produtos impressos em papel, noventa por cento dos erros dos pontos coletados no produto cartográfico/topográfico (ortoimagem), quando comparados com as suas coordenadas homólogas levantadas em campo por método de alta precisão, devem apresentar valores iguais ou inferiores aos previstos no Padrão de Exatidão Cartográfica dos Produtos Cartográficos Digitais (PEC-PCD). Também devem apresentar valores de erro médio quadrático (EMQ) ou erro padrão (EP) iguais ou inferiores aos previstos, conforme indicados nas Tabelas I, 2 e 3.

Na Tabela I, o PEC-PCD Planimétrico e o EP das classes " $B$ ", "C" e "D" correspondem, nessa ordem, as classes "A", "B", "C" do PEC Planimétrico previstas no Decreto $n^{\circ} 89.817$, de 20 de junho de 1984.

A Tabela 2 estabelece o PEC-PCD para os Modelos Digitais de Terreno (MDT), de Elevação (MDE) e de Superfície (MDS) e para os Pontos Cotados. Os valores previstos para a classe "A" (PEC-PCD) foram definidos a partir de adaptações dos estudos realizados por Merchant (1982) e ASPRS ( 1989), nos quais o PEC-PCD $=0,27^{*}$ Equidistância do produto cartográfico e o $E P=1 / 6$ *Equidistância do produto cartográfico. As classes " $B$ ", " $C$ " e "D" do PEC-PCD correspondem, em ordem, as classes "A", "B", "C" do PEC Altimétrico previstas no Decreto 89.817 , de 20 de junho de 1984.

A Tabela 3, o PEC-PCD Altimétrico e o EP das classes "A", " $B$ " e "C" correspondem, respectivamente, às classes " $A$ ", " $B$ " e " $C$ " do PEC Altimétrico previstas no Decreto 89.817, de 20 de junho de 1984.

Segundo Silva [5], um MDT ou MNT (Modelo Numérico de Terreno) pode ser definido como uma representação matemática computacional que descreve a superfície. As funções matemáticas empregadas são denominadas interpolações, e a superfície a ser apresentada é a superfície modelada. No caso deste estudo, foram gerados três MDTs.

Em superfícies tridimensionais, a modelagem consiste em determinar a superfície que descreve o comportamento de um conjunto de pontos definidos por suas coordenadas $(\mathrm{X}, \mathrm{Y}, \mathrm{H})$. As coordenadas $(\mathrm{X}, \mathrm{Y})$ são as variáveis independentes 
Tabela I. PEC-PCD Planimétrico [4]

\begin{tabular}{|c|c|c|c|c|c|c|c|c|c|c|c|c|c|c|c|c|c|}
\hline \multirow[b]{2}{*}{ PEC $^{(I)}$} & \multirow{2}{*}{ 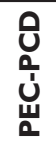 } & \multicolumn{2}{|c|}{ I:I.000 } & \multicolumn{2}{|c|}{$\mathrm{I}: 2.000$} & \multicolumn{2}{|c|}{$\mathrm{I}: 5.000$} & \multicolumn{2}{|c|}{$I: 10.000$} & \multicolumn{2}{|c|}{$I: 25.000$} & \multicolumn{2}{|c|}{$\mathrm{I}: 50.000$} & \multicolumn{2}{|c|}{$\mathrm{I}: 100.000$} & \multicolumn{2}{|c|}{$\mathrm{I}: 250.000$} \\
\hline & & $\begin{array}{c}\text { PEC } \\
\text { (m) }\end{array}$ & $\begin{array}{c}\text { EP } \\
(m)\end{array}$ & $\begin{array}{r}\text { PEC } \\
(\mathrm{m})\end{array}$ & $\begin{array}{c}\text { EP } \\
(m)\end{array}$ & $\begin{array}{r}\text { PEC } \\
(\mathrm{m})\end{array}$ & $\begin{array}{c}\text { EP } \\
(m)\end{array}$ & $\begin{array}{l}\text { PEC } \\
(\mathrm{m})\end{array}$ & $\begin{array}{l}\text { EP } \\
(m)\end{array}$ & $\begin{array}{r}\text { PEC } \\
\text { (m) }\end{array}$ & $\begin{array}{l}\text { EP } \\
(m)\end{array}$ & $\begin{array}{r}\text { PEC } \\
(\mathrm{m})\end{array}$ & $\begin{array}{c}\text { EP } \\
(m)\end{array}$ & $\begin{array}{c}\text { PEC } \\
(m)\end{array}$ & $\begin{array}{l}\text { EP } \\
(m)\end{array}$ & $\begin{array}{l}\text { PEC } \\
(\mathrm{m})\end{array}$ & $\begin{array}{l}\text { EP } \\
(m)\end{array}$ \\
\hline- & $A^{(2)}$ & & & & & & & 2,8 & & 7, & & 14 & & & & 70 & \\
\hline A & $\mathrm{B}^{(1)}$ & 50 & 0 & 1,00 & 0 & 2,50 & 1 & 5,00 & 3,00 & 12,50 & 7,50 & 25,00 & 00 & 50,00 & 30,00 & 125,00 & 75,00 \\
\hline B & $C^{(1)}$ & 0,80 & 0,50 & 1,60 & 1,00 & 4,00 & 2,50 & 8,00 & 5,00 & 20,00 & 12,50 & 40,00 & 25,00 & 80,00 & 50,00 & 200,00 & 125,00 \\
\hline C & (1) & 00 & 60 & 00 & 20 & 00 & 3,00 & 10,00 & 6,00 & 25,00 & 15,00 & 50,00 & 30,00 & 100,00 & 60,00 & 250,00 & 150,0 \\
\hline
\end{tabular}

Tabela 2. PEC-PCD para os Modelos Digitais de Terreno (MDT), de Elevação (MDE) e de Superfície (MDS) [4]

\begin{tabular}{|c|c|c|c|c|c|c|c|c|c|c|c|c|c|c|c|c|}
\hline \multirow{2}{*}{ 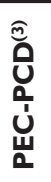 } & \multicolumn{2}{|c|}{$\begin{array}{c}\mathrm{I}: \mathrm{I} .000 \\
(\text { Eqd }=\mathrm{I} \mathrm{m})\end{array}$} & \multicolumn{2}{|c|}{$\begin{array}{c}\mathrm{I}: 2.000 \\
(\text { Eqd }=\mathrm{I} \mathrm{m})\end{array}$} & \multicolumn{2}{|c|}{$\begin{array}{c}\mathrm{l}: 5.000 \\
(\text { Eqd }=2 \mathrm{~m})\end{array}$} & \multicolumn{2}{|c|}{$\begin{array}{c}\mathrm{I}: \mathrm{I} 0.000 \\
(\text { Eqd }=5 \mathrm{~m})\end{array}$} & \multicolumn{2}{|c|}{$\begin{array}{c}\mathrm{I}: 25.000 \\
(\text { Eqd }=10 \mathrm{~m})\end{array}$} & \multicolumn{2}{|c|}{$\begin{array}{c}\mathrm{I}: 50.000 \\
(\text { Eqd }=20 \mathrm{~m})\end{array}$} & \multicolumn{2}{|c|}{$\begin{array}{c}\mathrm{I}: \mathrm{I} 00.000 \\
(\text { Eqd }=\mathbf{5 0} \mathrm{m})\end{array}$} & \multicolumn{2}{|c|}{$\begin{array}{c}\mathrm{I}: 250.000 \\
(\text { Eqd }=100 \mathrm{~m})\end{array}$} \\
\hline & & EP & & & & & & & & & & & & & & \\
\hline 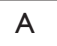 & & & & & & & & & & & & & & & & \\
\hline B & & & & & & & & & & & & & & & & \\
\hline C & & & & & & & 3,00 & & & & & & 30,00 & & 60,00 & \\
\hline$D$ & .75 & 0 & 0,75 & &, 50 & 1,00 & 3,75 & $2, J 0$ & 7,50 & 0,00 & 15,00 & 10,00 & 37,50 & 25,00 & 75,00 & 50,00 \\
\hline
\end{tabular}

Tabela 3. PEC-PCD Altimétrico [4]

\begin{tabular}{|c|c|c|c|c|c|c|c|c|c|c|c|c|c|c|c|c|c|}
\hline \multirow{3}{*}{ PEC(I) } & \multirow{3}{*}{ 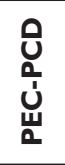 } & \multirow{2}{*}{\multicolumn{2}{|c|}{$\begin{array}{c}\mathrm{I}: \mathrm{I} .000 \\
(\text { Eqd }=\mathrm{I} \mathrm{m})\end{array}$}} & \multicolumn{2}{|c|}{$\mathrm{I}: 2.000$} & \multicolumn{2}{|c|}{$I: 5.000$} & \multicolumn{2}{|c|}{$I: 10.000$} & \multicolumn{2}{|c|}{$I: 25.000$} & \multicolumn{2}{|c|}{$I: 50.000$} & \multicolumn{2}{|c|}{$I: 100.000$} & \multicolumn{2}{|c|}{$\mathrm{I}: 250.000$} \\
\hline & & & & (Eqd & ( m) & (Eqc & $2 \mathrm{~m})$ & (Eqd & $5 \mathrm{~m})$ & (Eqd & $10 \mathrm{~m})$ & (Eqd & $20 \mathrm{~m}$ ) & (Eqd & $0 \mathrm{n}$ & (Eqd & $100 \mathrm{~m})$ \\
\hline & & $\begin{array}{l}\text { PEC } \\
(\mathrm{m})\end{array}$ & $\begin{array}{l}\text { EP } \\
(\mathrm{m})\end{array}$ & $\begin{array}{l}\text { PEC } \\
\text { (m) }\end{array}$ & $\begin{array}{l}\text { EP } \\
(\mathrm{m})\end{array}$ & $\begin{array}{l}\text { PEC } \\
\text { (m) }\end{array}$ & $\begin{array}{l}\text { EP } \\
(\mathrm{m})\end{array}$ & $\begin{array}{l}\text { PEC } \\
\text { (m) }\end{array}$ & $\begin{array}{l}\text { EP } \\
(\mathrm{m})\end{array}$ & $\begin{array}{l}\text { PEC } \\
(\mathrm{m})\end{array}$ & $\begin{array}{l}\text { EP } \\
(\mathrm{m})\end{array}$ & $\begin{array}{l}\text { PEC } \\
(\mathrm{m})\end{array}$ & $E P(m)$ & $\begin{array}{l}\text { PEC } \\
\text { (m) }\end{array}$ & $\operatorname{EP}(m)$ & $\begin{array}{l}\text { PEC } \\
(\mathrm{m})\end{array}$ & $\operatorname{EP}(m)$ \\
\hline$A$ & $A$ & 0,50 & 0,33 & 0,50 & 0,33 & 1,00 & 0,67 & 2,50 & 1,67 & 5,00 & 3,33 & 10,00 & 6,67 & 25,00 & 16,67 & 50,00 & 33,33 \\
\hline B & B & 0,60 & 0,40 & 0,60 & 0,40 & 1,20 & 0,80 & 3,00 & 2,00 & 6,00 & 4,00 & 12,00 & 8,00 & 30,00 & 20,00 & 60,00 & 40,00 \\
\hline C & $C$ & 0,75 & 0,50 & 0,75 & 0,50 & 1,50 & $\mathrm{I}, 00$ & 3,75 & 2,50 & 7,50 & 5,00 & 15,00 & 10,00 & 37,50 & 25,00 & 75,00 & 50,00 \\
\hline & $\mathrm{D}^{(4)}$ & 1,00 & 0,60 & 1,00 & 0,60 & 2,00 & $\mathrm{I}, 20$ & 5,00 & 3,00 & 10,00 & 6,00 & 20,00 & 12,00 & 50,00 & 30,00 & 100,00 & 60,00 \\
\hline
\end{tabular}

e $(H)$ a variável dependente, de forma que: $H=f(X, Y)$. $O$ valor da variável $(H)$ pode ser calculado para quaisquer valores de $(X, Y)$ pertencentes ao domínio da função. Para o caso de um modelo numérico de terreno, as funções de interpolação são usadas para determinar a altitude $\left(\mathrm{H}_{\mathrm{p}}\right)$ de um ponto $(P)$ qualquer, a partir de valores conhecidos das altitudes $\left(\mathrm{H}_{1}\right)$ de seus vizinhos.

Neste trabalho foi utilizado o SIFT, que é um algoritmo de visão computacional [6], capaz de transformar uma imagem em uma coleção de vetores de características locais. O SIFT é composto por duas partes distintas: o detector e o descritor. $O$ detector SIFT é baseado em cálculos de diferença de Gaussianas e o descritor SIFT utiliza histogramas de gradientes orientados para descrever a vizinhança local dos pontos de interesse. Mikolajczyk e Schimid [7] afirmam que o SIFT apresenta um desempenho superior em relação aos demais algoritmos estudados por eles em termos de invariância. Já Bauer [8] em seu artigo comparativo entre os algoritmos e suas variações, descreve aspectos positivos e negativos referente aos SIFT em relação SURF (Speeded Up Robust Features).

\section{MATERIAIS E MÉTODOS}

O estudo foi realizado na pedreira da empresa INCOPEL, localizada no município de Estância Velha, estado do Rio Grande do Sul. A área situa-se a aproximadamente $50 \mathrm{~km} \mathrm{da}$ capital do estado, Porto Alegre, e tem como acesso principal a Rodovia BR-I16, km 232. As coordenadas do centro geométrico da área (Sistema de Referência SIRGAS 2000) são as seguintes: $23^{\circ} 37^{\prime} 5 \mathrm{I} .07^{\prime \prime} \mathrm{S}$ e $5 \mathrm{I}^{\circ} 08^{\prime} 48.46^{\prime} \mathrm{O}$, altitude ortométrica média de $138 \mathrm{~m}$ e área aproximada de 12 ha.

O trabalho de obtenção de imagens foi realizado em janeiro de 2016 e dividido em duas etapas: o estabelecimento de Pontos de Controle (alvos na cor preta) e de Pontos Amostrais (alvos na cor vermelha) e o levantamento aerofotogramétrico (três voos).

A principal fonte de controle geométrico em fotogrametria são os pontos de controle destinados à amarração das aerofotos. Eles são essenciais para aerotriangulação para orientações das imagens. Como pontos de controle, foram utilizadas 9 placas de polietileno na cor branca, com um alvo central marcado na cor preta nas dimensões de $0,9 \mathrm{~m} \times 0,9 \mathrm{~m}$. Os alvos foram distribuídos nas 7 bancadas 
da pedreira em variadas altitudes. Após a instalação dos alvos, os mesmos foram levantados, utilizando-se a técnica RTK a partir de um conjunto GNSS-RTK da marca South composto de receptores base e móvel de dupla frequência modelo $586 \mathrm{~S}$ com precisão nominal horizontal e vertical, respectivamente, de $\pm 8 \mathrm{~mm}$ e $\pm 15 \mathrm{~mm}$, link de rádio base-móvel e demais acessórios. Os pontos amostrais foram utilizados como referência altimétrica para comparar com os modelos gerados na aerofotogrametria. As placas foram confeccionadas, nas dimensões $0,90 \mathrm{~m} \times 0,90 \mathrm{~m}$, com o material de polietileno na cor branca, com um alvo central marcado na cor vermelha.

Na pesquisa de campo foi utilizado o quadricóptero do modelo da DJI Phantom Pro, com as seguintes características: a) Sistema a FPV embutido, que transfere vídeo no seu smartphone (iOS / Android) através de Wi-Fi $(300 \mathrm{~m})$; b) Extensor Wi-Fi incluído, capaz de capturar até 980 pés acima; c) Gimbal motorizado, que controla o ângulo de inclinação da câmera e é manualmente controlável através do aplicativo Vision; d) Tempo de voo de aproximadamente 25 minutos com uma única carga, usando a bateria LiPo 4S $4.480 \mathrm{mAh}$; e) Receptor GNSS (GPS + GLONASS); f) Peso (incluindo a bateria) de I.280g.

O plano de voo foi realizado no software Pix 4D Capture, instalado em um smartfone com plataforma Android, utilizando como base os parâmetros do Phantom Pro e capaz de realizar voos autônomos remotamente controlados por meio do sistema de rádio e de rede Wifi própria.

Com base nas dimensões da área a serem levantadas, foram geradas automaticamente 9 faixas de voo, considerando $85 \%$ para sobreposição longitudinal e $60 \%$ para sobreposição lateral, conforme recomendações do fabricante do VANT para garantir a adequada sobreposição das imagens e, consequentemente, a qualidade do trabalho.

Foram realizados 3 voos com características idênticas: Altura do voo: $80 \mathrm{~m}$; Tempo aproximado de cada voo: 15 minutos; Número de faixas de voo: 9.

\section{RESULTADOS E DISCUSSÃO}

Após a prática das tomadas das aero imagens em campo, deu-se início ao processamento de dados e posteriormente foram gerados os MDTs. Os dados brutos da base foram submetidos ao serviço de Posicionamento por Ponto Preciso do IBGE. Em seguida, os pontos foram reprocessados com as novas coordenadas, possibilitando a montagem dos mosaicos das nove faixas e do fotoíndice.

O transporte de coordenadas da base do receptor GNSS foi realizado no IBGE-PPP que pode ser encontrado no site do IBGE. Este serviço de processamento é on-line para pós-processar dados GNSS, a um nível de confiança de $95 \%$, que permite aos usuários obterem coordenadas precisas no Sistema de Referência Geocêntrico para as Américas (SIRGAS 2000).

Após a realização do transporte de coordenadas, os pontos coletados em tempo real foram reprocessados no software FieldGenius, versão 8, utilizando a coordenada horizontal e a altitude ortométrica do relatório do PPP.

A Tabela 4 mostra os pontos de controle, desvios padrões (Sigma), calculados para cada eixo (N, E, Z) e resultados dos dados GNSS e a Tabela 5 mostra os pontos amostrais, desvios padrões, calculados para cada eixo (N, E, Z) e resultados dos dados GNSS.

As Tabelas 6, 7, 8 e 9 mostram os dados de coordenadas posicionais obtidos nos três levantamentos realizados, os respectivos MDTs, e os erros relacionados aos pontos amostrais de campo.

Conforme mencionado na introdução, para que um produto digital possa ser aceito como produto de Referência do Sistema Cartográfico Nacional (SCN) e, consequentemente, para a Infraestrutura Nacional de Dados Espaciais (INDE), é importante analisar os valores calculados do erro médio quadrático EMQxy e o EMQz dos pontos amostrais.

Para atender a PEC - PCD de forma fidedigna, foram calculados o EMQxy e EMQy dos três levantamentos, utilizando os pontos amostrais (alvos vermelhos). Analisando o EMQxy e o EMQz dos três voos (relatório do Photoscan), a partir

Tabela 4. Coordenadas dos pontos de controle

\begin{tabular}{|c|c|c|c|c|c|c|c|}
\hline Ponto & $\begin{array}{l}\text { SIRGAS } 2000 \\
\text { Norte (m) }\end{array}$ & Sigma (m) & $\begin{array}{c}\text { SIRGAS } 2000 \\
\text { Este (m) }\end{array}$ & Sigma (m) & $\begin{array}{c}\text { Altura } \\
\text { Ortométrica } \\
(\mathbf{m})\end{array}$ & $\begin{array}{c}\text { Altura } \\
\text { Elipsoidal (m) }\end{array}$ & Sigma (m) \\
\hline BASE & $6722|27,79|$ & 0,0020 & 485643,872 & 0,0080 & 105,970 & 110,88 & 0,0160 \\
\hline $\mathrm{PI}$ & 6722149,243 & 0,0010 & 485645,146 & 0,0017 & 105,870 & $\mathrm{II}, 780$ & 0,0030 \\
\hline P2 & 6722259,545 & 0,0041 & 485728,085 & 0,0066 & 127,589 & 133,499 & 0,0142 \\
\hline P3 & 6722227,61 & 0,0022 & 485790,699 & 0,0030 & 139,950 & 145,860 & 0,0067 \\
\hline P4 & $6722 \mid 47,733$ & 0,0024 & $485884, \mid 48$ & 0,0033 & 138.926 & 144,836 & 0,0081 \\
\hline P5 & 6722197,279 & 0,0023 & 485856,422 & 0,0029 & 147,074 & 152,984 & 0,0076 \\
\hline P6 & 6722223,704 & 0,0020 & 485872,656 & 0,0024 & $|60,90|$ & $|66,8| \mid$ & 0,0072 \\
\hline P7 & 6722282, II & 0,0039 & 485809,908 & 0,0046 & 165,682 & $|7|, 592$ & 0,0137 \\
\hline P8 & 6722079,44 & 0,0012 & 485734,311 & 0,0013 & 78,109 & 84,019 & 0,0053 \\
\hline P9 & 6722168,038 & 0,0016 & 485827,052 & 0,0018 & $|29,27|$ & $|35,18|$ & 0,0077 \\
\hline
\end{tabular}


Tabela 5. Coordenadas dos pontos amostrais

\begin{tabular}{|c|c|c|c|c|c|c|c|}
\hline Ponto & $\begin{array}{c}\text { SIRGAS } 2000 \\
\text { Norte (m) }\end{array}$ & $\begin{array}{c}\text { Sigma } \\
(\mathrm{m})\end{array}$ & $\begin{array}{l}\text { SIRGAS } 2000 \\
\text { Este }(\mathrm{m})\end{array}$ & $\begin{array}{c}\text { Sigma } \\
(\mathrm{m})\end{array}$ & $\begin{array}{l}\text { Altura Ortométrica } \\
(\mathrm{m})\end{array}$ & $\begin{array}{c}\text { Altura Elipsoidal } \\
(\mathrm{m})\end{array}$ & $\begin{array}{l}\text { Sigma } \\
(\mathrm{m})\end{array}$ \\
\hline BASE & $6722|27,79|$ & 0,0020 & 485643,872 & 0,0080 & 105,97 & 110,880 & 0,0160 \\
\hline VI & 6722072,059 & 0,0024 & 485669,762 & 0,0027 & 78,133 & 84,0430 & 0,0112 \\
\hline V2 & 6722141,137 & 0,0011 & 485703,142 & 0,0019 & 105,23 & $1 \mathrm{II}, 140$ & 0,0034 \\
\hline V3 & 6722182,573 & 0,0016 & $48575 I, 876$ & 0,0026 & 116,369 & 122,279 & 0,0047 \\
\hline V4 & 6722210,184 & 0,0024 & 485784,138 & 0,0037 & 128,629 & 134,539 & 0,008 I \\
\hline V5 & 6722221,874 & 0,0030 & 485799,873 & 0,0042 & $|39,56|$ & $|45,47|$ & 0,0103 \\
\hline V6 & 6722239,427 & 0,0024 & 485819,414 & 0,0030 & $|50,5| \mid$ & $|56,42|$ & 0,0076 \\
\hline V7 & 6722252,943 & 0,0128 & 485837,972 & 0,0150 & 162,405 & 168,315 & 0,0460 \\
\hline
\end{tabular}

Tabela 6. Erro Médio Quadrático Planimétrico calculados para o MDTI

\begin{tabular}{ccccc}
\hline Ponto & Este $(\mathbf{m})$ & Sigma $(\mathbf{m})$ & Norte $(\mathbf{m})$ & Sigma $(\mathbf{m})$ \\
\hline VI & 485669,931 & $-0,169$ & 6722072,008 & $0,05 I$ \\
V2 & 485703,208 & $-0,066$ & 6722141,144 & $-0,007$ \\
V3 & 485751,919 & $-0,043$ & 6722182,647 & $-0,074$ \\
V4 & 485784,188 & $-0,050$ & 6722210,206 & $-0,022$ \\
V5 & 485799,936 & $-0,063$ & 6722221,859 & 0,015 \\
V6 & 485819,409 & 0,005 & 6722239,418 & 0,009 \\
V7 & 485837,950 & 0,022 & 6722253,013 & $-0,070$ \\
\end{tabular}

Tabela 7. Erro Médio Quadrático Planimétrico calculados para o MDT2

\begin{tabular}{ccccc}
\hline Ponto & Este $(\mathbf{m})$ & Sigma $(\mathbf{m})$ & Norte $(\mathbf{m})$ & Sigma $(\mathbf{m})$ \\
\hline VI & 485670,015 & $-0,253$ & 6722072,006 & 0,053 \\
V2 & 485703,265 & $-0,123$ & 6722141,158 & $-0,021$ \\
V3 & 485751,902 & $-0,026$ & 6722182,703 & $-0,130$ \\
V4 & 485784,112 & 0,026 & 6722210,202 & $-0,018$ \\
V5 & 485799,940 & $-0,067$ & 6722221,865 & 0,009 \\
V6 & 485819,455 & $-0,041$ & 6722239,404 & 0,023 \\
V7 & 485838,006 & $-0,034$ & 6722252,96 & $-0,017$ \\
\end{tabular}

Tabela 8. Erro Médio Quadrático Planimétrico calculados para o MDT3

\begin{tabular}{ccccc}
\hline Ponto & Este $(\mathbf{m})$ & Sigma $(\mathbf{m})$ & Norte $(\mathbf{m})$ & Sigma $(\mathbf{m})$ \\
\hline VI & 485669,949 & $-0,187$ & 6722072,02 & 0,039 \\
V2 & 485703,262 & $-0,120$ & 6722141,09 & 0,047 \\
V3 & 485751,900 & $-0,024$ & 6722182,731 & $-0,158$ \\
V4 & 485784,132 & 0,006 & 6722210,243 & $-0,059$ \\
V5 & 485799,927 & $-0,054$ & 6722221,875 & $-0,001$ \\
V6 & 485819,415 & $-0,001$ & 6722239,42 & 0,007 \\
V7 & 485837,960 & 0,012 & 6722253,022 & $-0,079$ \\
\end{tabular}

Tabela 9. Erro Médio Quadrático Altimétrico

\begin{tabular}{cccc}
\hline Pontos & MDTI & MDT2 & MDT3 \\
\hline VI & 0,063 & 0,006 & 0,033 \\
V2 & $-0,010$ & $-0,024$ & $-0,002$ \\
V3 & $-0,018$ & 0,008 & $-0,008$ \\
V4 & $-0,058$ & $-0,097$ & $-0,053$ \\
V5 & $-0,002$ & $-0,004$ & $-0,037$ \\
V6 & $-0,076$ & $-0,036$ & $-0,055$ \\
V7 & 0,032 & 0,026 & $-0,024$ \\
EMQSZ & $\mathbf{0 , 0 4 5 6 m}$ & $\mathbf{0 , 0 4 1 5 m}$ & $\mathbf{0 , 0 3 5 m}$ \\
\hline
\end{tabular}


dos dados observa-se que os mesmos ficaram bem abaixo do solicitado no PEC - PCD Planimetria (Classe A: 0,28m) e PEC - PCD Altimétria (Classe A: $0,50 \mathrm{~m}$ ), portanto atenderiam a escala de $1 / 1000$ categoria "A" previstas no Decreto 89.817, de 20 de junho de 1984.

As imagens foram processadas, no software Agsoft Photoscan, que utiliza o algoritmo SIFT, e foi desenvolvido para realizar operações de fotogrametria em imagens e extração de informações. $O$ mesmo possibilita trabalhar com imagens a partir de uma ampla variedade de fontes e formatos, controle de solo, orientação e dados GNSS. O software Photoscan converte imagens aéreas em mosaicos 2D e 3D georrefenciados, utilizando pontos de apoio de GCP (pontos de controle de solo) para geo-localização e estimação da elevação ortométrica dos 7 pontos amostrais. Estes dados são apresentados na Tabela 10.

Nas Tabelas I I a |4, é possível observar as discrepâncias encontradas entre Altitudes Ortométricas GNSS x Altitudes Ortométricas medidas nos MTDs.

Para complementar o cálculo da acurácia do modelo foi realizada uma análise com base nas discrepâncias e nos fatores de correlação, de forma a permitir o enquadramento do produto final de acordo com a legislação pertinente bem como em relação às necessidades práticas dos profissionais envolvidos com cálculos de volumes em minas. Na Tabela I5, é possível observar as médias encontradas Altitude Ortométrica medidas nos 3 modelos.

Através da análise das discrepâncias encontradas nas Tabelas II, 12 e I3, foi possível observar as diferenças entre os modelos, que variaram entre $-0,076 \mathrm{~m}$ até $+0,063 \mathrm{~m}$ no MDTI, $-0,097 \mathrm{~m}$ até $+0,026 \mathrm{~m}$ no MDT2 e o $-0,055 \mathrm{~m}$ até $+0,033 \mathrm{~m}$ no MDT3. Pode-se perceber que as médias encontradas nos modelos, respectivamente, foram de: $-0,010,-0,017$ e - 0,021 metros, permitindo acurácia decimétrica, com benefícios em relação aos métodos convencionais, como eficiência e, principalmente, rapidez.

$\mathrm{Na}$ Tabela 16, é possível observar os coeficientes de Correlação das duas variáveis, Altitude Ortométrica Digital x Acurácia dos três modelos, calculados no software Excel 2013. Em estatística descritiva, o coeficiente de correlação de Pearson tem como objetivo medir o grau da correlação e dar a direção entre duas variáveis de escala métrica, classificando como positiva ou negativa.

Este coeficiente, normalmente representado por $p$ assume apenas valores entre $-\mathrm{I}$ e $\mathrm{I}$.

- $p=$ I Significa uma correlação perfeita positiva entre as duas variáveis.

- $p=-$ I Significa uma correlação negativa perfeita entre as duas variáveis - Isto é, se uma aumenta, a outra sempre diminui.

- $p=0$ Significa que as duas variáveis não dependem linearmente uma da outra. No entanto, pode existir uma dependência não linear.

Observando a Tabela 16, conclui-se que o grau de correlação no MDTI é negativo fraco, já as correlações do MTD2 e MDT3, são positivas fracas, comprovando que não dependem linearmente uma da outra, ou seja, a altitude no experimento não está relacionada diretamente à acurácia altimétrica.

Tabela 10. Altitudes medidas no ortomosaico gerados no software Photoscan

\begin{tabular}{cccc}
\hline Alvo & $\begin{array}{c}\text { Altura Ortométrica }(\mathbf{m}) \\
\text { MDTI }\end{array}$ & $\begin{array}{c}\text { Altura Ortométrica }(\mathbf{m}) \\
\text { MDT2 }\end{array}$ & $\begin{array}{c}\text { Altura Ortométrica }(\mathbf{m}) \\
\text { MDT3 }\end{array}$ \\
\hline VI & 78,196 & 78,139 & 78,166 \\
V2 & 105,220 & 105,206 & 105,228 \\
V3 & $116,35 \mid$ & 116,377 & 116,369 \\
V4 & $128,57 \mid$ & 128,532 & 128,576 \\
V5 & 139,559 & 139,557 & 139,524 \\
V6 & 150,435 & 150,475 & 150,456 \\
V7 & 162,437 & $162,43 \mid$ & $162,38 \mid$ \\
\hline
\end{tabular}

Tabela I I. Discrepâncias GNSS x MDTI

\begin{tabular}{cccc}
\hline Alvo & $\begin{array}{c}\text { Altitude Ortométrica }(\mathbf{m}) \\
\text { GNSS }\end{array}$ & $\begin{array}{c}\text { Altitude Ortométrica }(\mathbf{m}) \\
\text { MDT }\end{array}$ & Discrepância (m) \\
\hline VI & 78,133 & 78,196 & $+0,063$ \\
V2 & 105,230 & 105,220 & $-0,010$ \\
V3 & 116,369 & 116,351 & $-0,018$ \\
V4 & 128,629 & $128,57 \mid$ & $-0,058$ \\
V5 & $139,56 \mid$ & 139,559 & $-0,002$ \\
V6 & 150,511 & 150,435 & $-0,076$ \\
V7 & 162,405 & 162,437 & $+0,032$ \\
\hline
\end{tabular}


Tabela 12. Discrepâncias GNSS x MDT2

\begin{tabular}{cccc}
\hline Alvo & Altitude Ortométrica $(\mathbf{m})$ GNSS & Altitude Ortométrica (m) MDT2 & Discrepância (m) \\
\hline VI & 78,133 & 78,139 & $+0,006$ \\
V2 & 105,230 & 105,206 & $-0,024$ \\
V3 & 116,369 & 116,377 & $+0,008$ \\
V4 & 128,629 & 128,532 & $-0,097$ \\
V5 & 139,561 & 139,557 & $-0,004$ \\
V6 & 150,511 & 150,475 & $-0,036$ \\
V7 & 162,405 & 162,431 & $+0,026$ \\
\hline
\end{tabular}

Tabela 13. Discrepâncias GNSS x MDT3

\begin{tabular}{cccc}
\hline Alvo & Altitude Ortométrica $(\mathbf{m})$ GNSS & Altitude Ortométrica (m) MDT3 & Discrepância (m) \\
\hline VI & 78,133 & 78,166 & $+0,033$ \\
V2 & 105,230 & 105,228 & $-0,002$ \\
V3 & 116,369 & 116.361 & $-0,008$ \\
V4 & 128,629 & 128,576 & $-0,053$ \\
V5 & 139,561 & 139,524 & $-0,037$ \\
V6 & 150,511 & 150,456 & $-0,055$ \\
V7 & 162,405 & 162,381 & $-0,024$ \\
\hline
\end{tabular}

Tabela 14. Média das discrepâncias

\begin{tabular}{cccc}
\hline Alvo & Discrepância $(\mathbf{m})$ MDTI & Discrepância $(\mathbf{m})$ MDT2 & Discrepância (m) MDT3 \\
\hline VI & 0,063 & 0,006 & 0,033 \\
V2 & $-0,010$ & $-0,024$ & $-0,002$ \\
V3 & $-0,018$ & 0,008 & $-0,008$ \\
V4 & $-0,058$ & $-0,097$ & $-0,053$ \\
V5 & $-0,002$ & $-0,004$ & $-0,037$ \\
V6 & $-0,076$ & $-0,036$ & $-0,055$ \\
V7 & 0,032 & 0,026 & $-0,024$ \\
Média & $-\mathbf{0 , 0 1 0}$ & $-\mathbf{0 , 0 1 7}$ & $-\mathbf{0 , 0 2 1}$ \\
\hline
\end{tabular}

Tabela 15. Médias de Altitude Ortométricas

\begin{tabular}{|c|c|c|c|c|}
\hline Alvo & $\begin{array}{c}\text { Altitude } \\
\text { Ortométrica(m) MDT I }\end{array}$ & $\begin{array}{c}\text { Altitude } \\
\text { Ortométrica(m) MDT2 }\end{array}$ & $\begin{array}{l}\text { Altitude Ortométrica } \\
\text { (m) MDT3 }\end{array}$ & Média (m) \\
\hline $\mathrm{VI}$ & 78,196 & 78,139 & 78,166 & 78,167 \\
\hline $\mathrm{V} 2$ & 105,22 & 105,206 & 105,228 & 105,217 \\
\hline V3 & $|16,35|$ & 116,377 & 116,361 & 116,363 \\
\hline V4 & $|28,57|$ & 128,532 & 128,576 & 128,560 \\
\hline V5 & 139,559 & 139,557 & 139,524 & 139,547 \\
\hline V6 & 150,435 & 150,475 & 150,456 & 150,455 \\
\hline V7 & 162,437 & $|62,43|$ & $|62,38|$ & $162,4 \mid 6$ \\
\hline
\end{tabular}

Tabela 16. Coeficiente de Correlação

\begin{tabular}{cc}
\hline Modelo & Coeficiente \\
\hline MDTI & $-0,2787$ \\
MDT2 & 0,2335 \\
MDT3 & 0,3403 \\
\hline
\end{tabular}

\section{CONCLUSÕES}

Conforme a Comissão Nacional de Cartografia (CONCAR), no que diz respeito às especificações técnicas de dados geoespaciais vetoriais, os resultados dos três 
levantamentos se enquadram na escala cartográfica de I:1000, categoria "A". Analisando EMQxy e EMQz, observou-se que $100 \%$ dos erros dos pontos coletados no produto cartográfico/topográfico (ortoimagem), quando comparados com as suas coordenadas levantadas em campo por método diferencial em tempo real, ficaram abaixo dos $0,28 \mathrm{~m}$, limite este solicitado pelo PEC - PCD planimetria e PEC - PCD altimetria previstos no Decreto 89.8I7, de 20 de junho de 1984.
Para o Departamento Nacional de Produção Mineral (DNPM), a referência de precisão e acurácia nos produtos cartográficos é a do CONCAR, portanto a escala encontrada no experimento satisfaz a norma e atende a finalidade de cálculo de volumes em cavas de minas.

O vento influenciou diretamente na acurácia planialtimétrica, causando turbulência durante a tomada das imagens. Tal fator é relevante na utilização desta tecnologia, portanto para futuros trabalhos sugere-se que seja avaliada a velocidade do vento no momento da coleta.

\section{REFERÊNCIAS}

I Jorge LAC. Metodologia para utilização de aeromodelos em monitoramento aéreo: análise de imagens. São Carlos: EMBRAPA Instrumentação Agropecuária; 2003. (Circular Técnica 18 de Novembro de 2003).

2 Slompo, PJH. Utilização de um veículo aéreo não tripulado para obtenção de dados dendrométricos de eucalyptus benthamii maiden et cambag [dissertação]. Garapuava: Universidade Estadual do Centro-Oeste; 2013.

3 Brito J, Coelho L. Fotogrametria Digital. Rio de Janeiro: Instituto Militar de Engenharia; 2002.

4 Comissão Nacional de Cartografia. Especificação técnica para a aquisição de dados geoespaciais vetoriais. infraestrutura nacional de dados espaciais. 2. ed. Rio de Janeiro: Exército Brasileiro; 201 I.

5 Silva I, Segantine PCL. Topografia para engenharia: teoria e prática de geomática. São Paulo: Campus; 2015.

6 Lowe DG. Distinctive Image Features from Scale Invariant. International Journal of Computer Vision. 2004;60:91110.

7 Mikolajczyk K, Schmid C. Indexing based on scale invariant interest points. In: International Conference on Computer Vision; July 200 I; Vancouver, Canadá. Piscataway: IEEE; 200 I. p. 525-53I.

8 Bauer J, Sünderhauf N, Protzel P. Comparing several implementations of two recently published feature detectors. IFAC Proceedings Volumes. 2007;40(I5): I43-I48.

Recebido em: 21 Mar. 2017

Aceito em: 22 Ago. 2017 